\title{
Bi-phase emulsion droplets as dynamic fluid optical systems
}

\author{
Sara Nagelberg ', Amy Goodling ${ }^{2}$, Kaushikaram Subramanian ${ }^{3}$, George Barbastathis ${ }^{1}$, Moritz Kreysing ${ }^{3}$, \\ Tim Swager ${ }^{4}$ Lauren Zarzar ${ }^{2}$, and Mathias Kolle ${ }^{1, *}$ \\ ${ }^{1}$ Department of Mechanical Engineering, Massachusetts Institute of Technology, Cambridge, MA, USA. \\ ${ }^{2}$ Department of Materials Science and Engineering, The Pennsylvania State University, University Park, PA, USA. \\ ${ }^{3}$ Max Planck Institute of Molecular Cell Biology and Genetics, Dresden, Germany. \\ ${ }^{4}$ Department of Chemical Engineering, Massachusetts Institute of Technology, Cambridge, MA, USA.
}

\begin{abstract}
Micro-scale optical components play a critical role in many applications, in particular when these components are capable of dynamically responding to different stimuli with a controlled variation of their optical behavior. Here, we discuss the potential of micro-scale bi-phase emulsion droplets as a material platform for dynamic fluid optical components. Such droplets act as liquid compound micro-lenses with dynamically tunable focal lengths. They can be reconfigured to focus or scatter light and form images. In addition, we discuss how these droplets can be used to create iridescent structural color with large angular spectral separation. Experimental demonstrations of the emulsion droplet optics are complemented by theoretical analysis and wave-optical modelling. Finally, we provide evidence of the droplets utility as fluidic optical elements in potential application scenarios.
\end{abstract}

\section{Bi-phase emulsion droplets with reconfigurable internal interface}

Micrometre-scale optical elements have contributed significantly to the miniaturization of optical devices and instrumentation [1-3]. To this end, optofluidic devices using dynamic fluid lens materials represent an ideal platform to create versatile, reconfigurable, refractive optical components [4]. Droplets smaller than the capillary length, wherein surface tension is the dominant force, create curved interfaces between fluid volumes [5]. These curved interfaces can be used to reflect and refract light forming tunable fluid micro-lenses, as individual components or arranged in arrays, for applications in miniaturized optical components and optics-based biosensing devices $[8,9]$. Here, we present a strategy to form fluidic tunable compound micro-lenses from stable hydrocarbon and fluorocarbon emulsion droplets in aqueous media [10]. We use combinations of transparent fluorocarbon fluids, such as perfluorohexane or Fluorinert FC-770 ( $\mathrm{n}=1.27)$, and hydrocarbons, such as heptane $(\mathrm{n}=1.39)$ or hexane $(\mathrm{n}=1.38)$. These bi-phase emulsion droplets can be dynamically reconfigured between double emulsion and Janus (two-sided) morphologies through application of external stimuli, including chemical triggers, light, and heat, which makes them very promising as highly tunable compound lenses (Fig. 1).

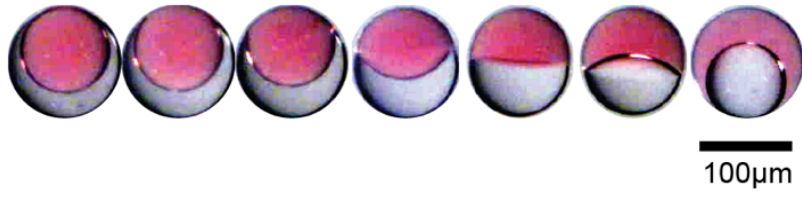

Fig. 1. Side view optical micrographs of droplets composed of FC-770 (grey) and heptane (red) with varying internal interface curvature. The red color of the heptane phase results from the incorporation of a Rhodamine-based dye.

\section{Bi-phase droplets as tunable lenses}

Due to their curved surfaces and interfaces and the refractive index contrast of the droplet constituents and the aqueous medium the droplets can function as lenses. Surfactants can be used to modify the relative interfacial tensions between the droplet phases and the surrounding medium to shape the droplet internal interfaces and thereby alter their lensing characteristics, such as the focal length (Fig. 2). In the presentation, we discuss potential application scenarios for such reconfigurable lenses in displays and 3D micro-imaging devices.

\footnotetext{
* Corresponding author: mkolle@mit.edu
} 

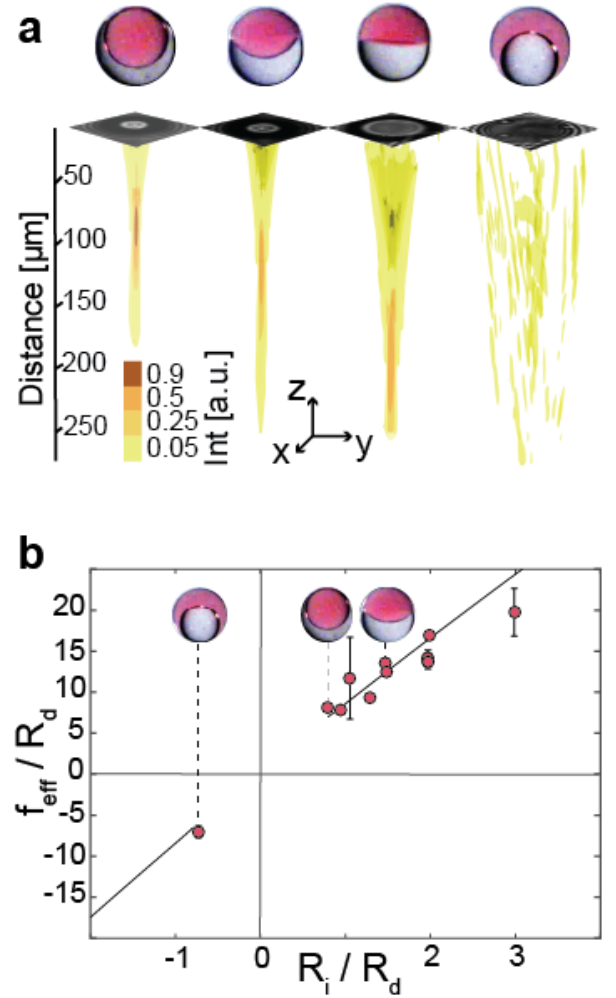

Fig. 2. a) Iso-surfaces of the reconstructed light fields behind the bi-phase droplets for different morphologies. b) Effective focal length as a function of internal radius of curvature $\mathrm{R}_{\mathrm{i}}$, normalized by the droplet diameter $\mathrm{R}_{\mathrm{d}}$.

\section{Color generation in bi-phase emulsion droplets}

In addition to light focusing, imaging, and beam steering, these fluid droplets can also be employed to create iridescent structural colors by cascaded total internal reflection and interference. Colors can generally result from many physical phenomena, including spectrally selective light absorption by pigments and dyes, materialspecific optical dispersion and light interference in microand nanometre-scale periodic structures. Scattering, diffraction and interference mechanisms are particularly relevant to spherical droplets, which contribute to atmospheric phenomena such as glories, coronas and rainbows [11, 12]. The bi-phase emulsion droplets support a previously unrecognized mechanism for creating iridescent structural color with large angular spectral separation. The effect relies on light travelling along different trajectories of total internal reflection at a concave optical interface, causing interfere that results in brilliant color patterns. Each droplet acts as a spatiospectral filter of incident light independent of other droplets in its surroundings (Fig. 3a). The color separation effect strongly depends on droplet size and morphology providing several different handles for controlling the phenomenon (Fig. 3b). A detailed characterization of the droplet colors, accompanied by theoretical modeling will be presented
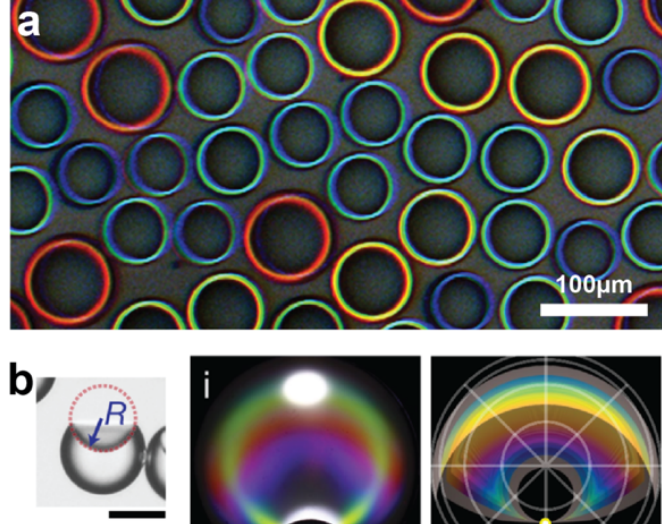

$R=2 \overline{4 \mu \mathrm{m}}$
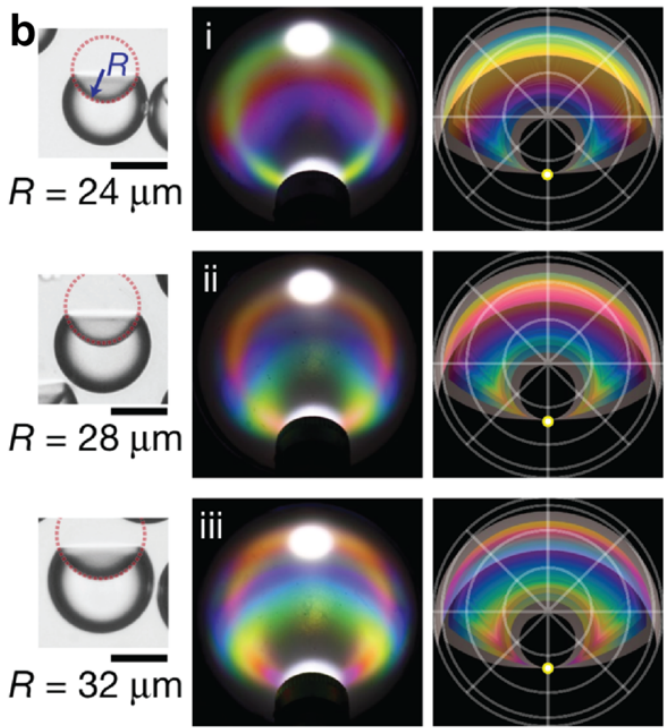

Fig. 3. a) Poly-disperse bi-phase emulsion droplets showing a size dependence of their color. b) Comparison of experimental iridescence maps of index-matched Janus droplets with the predicted three-dimensional calculation for various sizes.

\section{References}

1. Erdmann, L. \& Gabriel, K. J., Appl. Opt. 40, 55925599 (2001).

2. Martinez-Cuenca, R., Saavedra, G., Martinez-Corral, M. \& Javidi, B., J. Disp. Technol. 1, 321-327 (2005).

3. Xiao, X., Javidi, B., Martinez-Corral, M. \& Stern, A., Appl. Opt. 52, 546-560 (2013).

4. Hawkins, A. R. \& Schmidt, H., Handbook of Optofluidics (CRC Press, 2010).

5. Ren, H., Xu, S. \& Wu, S.-T., Opt. Commun. 283, 3255-3258 (2010).

6. Kim, J., Singh, N. \& Lyon, L. A., Angew. Chem. Int. Ed. 45, 1446-1449 (2006).

7. 41. Kuswandi, B., Nuriman, Huskens, J. \& Verboom, W., Anal. Chim. Acta 601, 141-155 (2007).

8. Zarzar, L. D. et al., Nature 518, 520-524 (2015).

9. Hulst, H.C. Light Scattering by Small Particles (Courier Corporation, Mineola, 1981).

10. Nussenzveig, H. M., J. Math. Phys. 10, 125-176 (1969). 